\title{
Review of the Book "Causality and Reversibility in Irreversible Time”
}

\author{
A. N. Morozov \\ Physics Department, Bauman Moscow State Technical University, Moscow, Russia \\ Email: amor59@mail.ru
}

Received January 19, 2012; revised February 23, 2012; accepted March 4, 2012

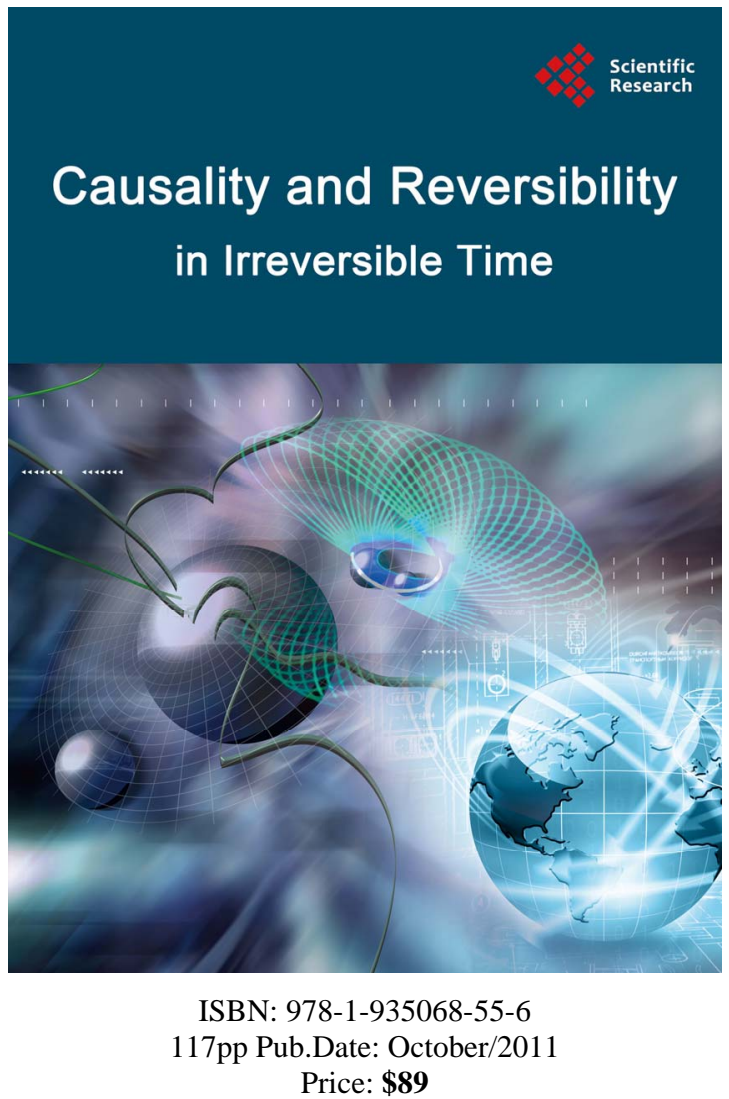

The book concerns a number of the most fundamental concepts and problems of modern physics: causality, entanglement and the nature of time. A distinguishing feature of author's approach is equal attention to both the theoretical and experimental challenges posed by those issues. From very beginning, in Introduction the author points out Kozyrev causal mechanics, suggested about fifty years ago, as an inspirable source of ideas developed him at the modern level of rigor.

Indeed, the progress in quantum mechanics has shed a new light on Kozyrev ideas and his experimental results. The possibility of observation of the future states as the existing reality demonstrated by the scientist seems now real. The quantum mechanical principle of weak causal- ity, originally qualitatively suggested by Cramer and then strictly formulated by Korotaev, (Chapter 3) admits availability of the signals in reverse time for the random processes. The series of experiments (Chapter 6) has revealed availability of the advanced response in the lab detectors to large-scale dissipative heliogeophysical processes. The high level of advanced correlation and the large time shift allowed to do the long-term forecasts of geomagnetic and solar activity.

The author goes to these final results step-by-step through the book. In Chapter 1 he considers direct development of Kozyrev semiclassical theory and its geophysical application. As a result a number of well know facts of planetary North-South asymmetry which seemed occasional before turns out quite regular.

In Chapter 2 the author reconsider starting points of causal mechanics and develops a new method of causal analysis at the beginning at the classical level. The basic idea of causal analysis lies in formalization of intuitive understanding of the asymmetry of a cause and an effect owing to which one can usually distinguish them without measuring of retardation. In other words the essence of causal analysis is based on formalization of cause and effect concepts from informational asymmetry of the processes without resort to time relation. The retardation of effect relative to the cause variables is introduced after their definition as an axiom. Although the causal analysis was first suggested by Korotaev in 1989 for solving of fundamental problems (and in such a manner it is described in the book) nowadays his method is employed by different researchers as a very practical tool of experimental data processing.

Next in Chapter 3 quantum causal analysis is considered. Somebody believes that the quantum world is causeless. It proved to be a particular case. Quantum entangled states can be causal and this causality is very interesting. The outstanding distinction of quantum causality from classical one is that the former can exist only in the mixed states. In other words finite quantum causality is possible only in the open systems. Moreover quantum causality admits time reversal, the consequences of which are con- 
sidered in the following chapters. In Chapter 3 causal analysis application is demonstrated by the wide series of entangled states. Again, although quantum causal analysis was suggested in connection with a basic problem of time, its application proves to be quite practical. For instance the interesting interrelations between causality and decoherence are discovered, which can be used in the entanglement protection tasks.

The Chapter 4 represents a theoretical-heuristic step to the macroscopic experiment. In spite of the recent progress, development of successive theory of macroscopic entanglement (which has to resemble classical thermodynamics, i.e. to operate with the macroscopic parameters) is difficult prolem and such a theory is absent at present. The author, leaning on action-at-a-distance electrodynamics and on the known fact of constructive role of dissipation in entanglement generation, suggests a reasonable equation of macroscopic entanglement. The equation predicts both retarded and advanced correlation and admits the experimental verification which is done in the last chapter. The model verification author demonstrates as soon as the equation has been introduced. At last Belltype inequality is derived, violation of which is an evi- dence of nonlocal nature of predicted macroscopic correlation.

In Chapter 5 the experimental approach to the detection of above correlations is carefully discussed. The theories of different detectors are described and various sources of interferences and errors are analyzed.

At last in Chapter 6 the results of wide series of longterm experiments are presented. These results are surprising. Really nonlocal (quantum) correlation under some conditions can be measured at the macro-level. And really time reversal causal connection is observed. Moreover the observed advanced correlation can be utilized for the random processes forecasting. Due to careful attention paid by the author to cleanness of the experiment these unusual results are convicting.

I think the book will attract widespread interest in basic physics community.

\section{REFERENCE}

[1] S. M. Korotaev, "Causality and Reversibility in Irreversible Time,” Scientific Reseach Publishing, USA, 2011.

\section{To order: http://www.scirp.org/book/}

\section{bookorder@scirp.org}

